\title{
The Effects of High and Low Dose Nicotine Administration on Neurogenesis
}

\author{
Soycan Mizrak*1, Mahmud Mustafa Özkut ${ }^{2}$, Sevinç Manisa İnan ${ }^{3}$ and Gulinnaz Ercan ${ }^{4}$ \\ ${ }^{1}$ Clinical Biochemistry Laboratory, Usak Training and Research Hospital, Usak, Turkey \\ ${ }^{2}$ Department of Histology and Embryology, Yozgat Bozok University, Yozgat, Turkey \\ ${ }^{3}$ Department of Histology and Embryology, Faculty of Medicine, Izmir University of Economics, Izmir, Turkey \\ ${ }^{4}$ Department of Medical Biochemistry, Faculty of Medicine, Ege University, Izmir, Turkey
}

Received: 制: December 28, 2018; Published: 制: January 10, 2019

*Corresponding author: Soycan Mizrak, Clinical Biochemistry Laboratory, Turkey and Gulinnaz Ercan, Department of Medical Biochemistry, Faculty of Medicine, Turkey

\begin{abstract}
Various pharmacological doses of nicotine during gestation have been examined and nicotine exposure alone is expected to be less harmful to the fetus than cigarette smoke. Nicotine affects the neurochemical systems. Neurotrophins are very important factors for neuronal development, and plasticity of neurons. In this study, considering the role of the hippocampus in both memory and learning, we investigated the effects of high and low dose nicotine administration in pre-postnatal periods, on neurogenesis with immunohistochemical methods. Brain Derived Neurotrophic Factor (BDNF), Neuronal Specific Nuclear Protein (NeuN) and S-100 protein were analysed to evaluate the neurons and neuroglial cells in the Swiss Albino rat hippocampus. Immunoreactivity scores were determined by using the semi-quantitative method. The intensity of immunoreactivity was evaluated as mild (+), moderate (++) or strong (+++) and the results were compared via using the ANOVA statistical test. As a result, BDNF, NeuN and $\mathrm{S}-100$ immunoreactivities of neurons in the hippocampus were found to have increased via nicotine administration. These factors can contribute to nicotine's neuroprotective effects. Nicotine exposure can enrich the reduced number of neurons or interneuron connections which decrease with aging, but more clinical studies are needed in this area to shed light the role of nicotine on neurogenesis.
\end{abstract}

\section{Introduction}

Tobacco is the most important substance abuse during pregnancy. About $20 \%$ of pregnant women smoke during their pregnancy. While pregnant women smoke, the developing fetus is exposed to nicotine, the main psychoactive component of tobacco and tobacco smoke ingredients [1,2]. Various pharmacological doses of nicotine during gestation have been tested and nicotine exposure alone is expected to be less harmful to the fetus than cigarette smoke [3]. Nicotine effects the dopaminergic and cholinergic neurochemical systems and alters the physiological and behavioural situations in the offspring [4]. During the development of the brain, neurotrophins are very important factors for neuronal plasticity proliferation, survival, development, and plasticity of neurons. One of the members of the neurotrophin family is BrainDerived Neurotrophic Factors (BDNF). BDNF influences GABAergic neuronal phenotype [5]; regulates the efficacy of GABAergic synapses [6].

The other specific protein of neurons is the neuronal specific nuclear protein (NeuN). Monoclonal antibodies to the NeuN protein is important for neuronal differentiation. The normal and pathologic functions of neurons are evaluated by using this marker [7]. The other one of the specific protein is $\mathrm{S} 100$ protein. These proteins are called S100 because of their solubility in a 100\%-saturated solution with ammonium sulfate at neutral $\mathrm{pH}$ [8]. These proteins are produced by astrocytes in the Central Nervous System (CNS). S100 protein's functions are regulation of proliferation, differentiation, apoptosis, Ca2 + homeostasis, energy metabolism and inflammation within cells [9]. In the present study, we investigated the effects of high and low dose nicotine administration on neurogenesis with immunohistochemical methods. Brain Derived Neurotrophic Factor (BDNF), Neuronal Specific Nuclear Protein (NeuN) and S-100 protein were analysed to evaluate the neurons and neuroglial cells in the rat hipocampus.

\section{Materials and Methods}

In this study, approved by the Animal Ethics Committee of Ege University School of Medicine (Bornova, Izmir, Turkey), the off springs of Swiss Albino rats were used. They were housed in 
a temperature-controlled room with a 12-hour light/dark cycle and maintained on standard laboratory animal chow and provided water ad libitum. All animals used in this study were maintained in accordance with the guidelines for animal welfare. Firstly, 10 female Swiss Albino rats were divided into 2 main groups; as a nicotine group $(n=5)$, and a control group $(n=5)$. They were conceived by adding 2 male rats to each group. While the control group was given normal drinking water, $0.4 \mathrm{mg} / \mathrm{kg}$ BW-body weight nicotine was prepared freshly every day and added to the nicotine group's drinking water. After birth, the off springs were left in the same cage with their mothers and fed through lactation. At the end of the sixth week as soon as the pups started to normal feeding, the pups in the nicotine group were divided split into 2 groups; one to be subjected to low, and one to high doses of nicotine (Low dose nicotine-LDN, 0.4mg nicotine/kg BW/day; High dose nicotine-HDN, $6.0 \mathrm{mg}$ nicotine/kg BW/day). In this way, we formed 3 groups; as control $(n=10), \operatorname{LDN}(n=10)$ and HDN $(n=10)$ groups. At the end of the 12th month, rats were sacrificed under general anesthesia (ketamine $75 \mathrm{mg} / \mathrm{kg}$ ve xylazine $10 \mathrm{mg} / \mathrm{kg}$ i.p.) and their craniums were dissected so that their brain samples could be taken out.

The right brain samples containing the hippocampus were fixed in $10 \%$ formaline solution and were prepared according to the routine paraffin tissue protocol and were embedded in paraffin. Five $\mu \mathrm{m}$ serial sections were taken on the polylisine coated lams and were stained histochemically with Hematoxylin-Eosin staining. After histological evaluation, serial sections, including the hippocampus were stained by using the immunohistochemical technique. In the samples, anti-BDNF, antiNeuN, anti-calbindin, anti-S100 and anti-GFAP primary antibodies were used to determine whether nicotine exposure had any effect on the immunohistochemical distributions of these antibodies on neurons and neuroglial cells. Immunoreactivity scores were determined by using the semi-quantitative method. The intensity of immunoreactivity was evaluated as mild $(+)$, moderate $(++)$ or strong $(+++)$ and the results were compared via using the ANOVA statistical test.

\section{Results}

Hippocampus samples of study groups in paraffin blocks were stained with H\&E and the molecular, pyramidal and polymorphic stages of the hippocampus were investigated under Olympus microscope (Olympus, Center Valley, PA) (Figure 1). Micrograph of control, $\mathrm{N}$ and $\mathrm{S}$ group's neurons were active and included euchromatic nucleus. It was observed that whether the intense of BDNF immunoreactivities in the control group's neurons were mild/moderate $(+/++)$, the intense of BDNF immunoreactivities in nicotine group's were moderate/strong $(++/+++)$ (Figure 2). It was observed that whether the intense of Neuron Specific Neuronal Protein (NeuN) immunoreactivities in the control group's neurons were mild/moderate $(+/++)$, the intense of NeuN immunoreactivities in nicotine group's were moderate $(++)$ (Table 1) (Figure 3).

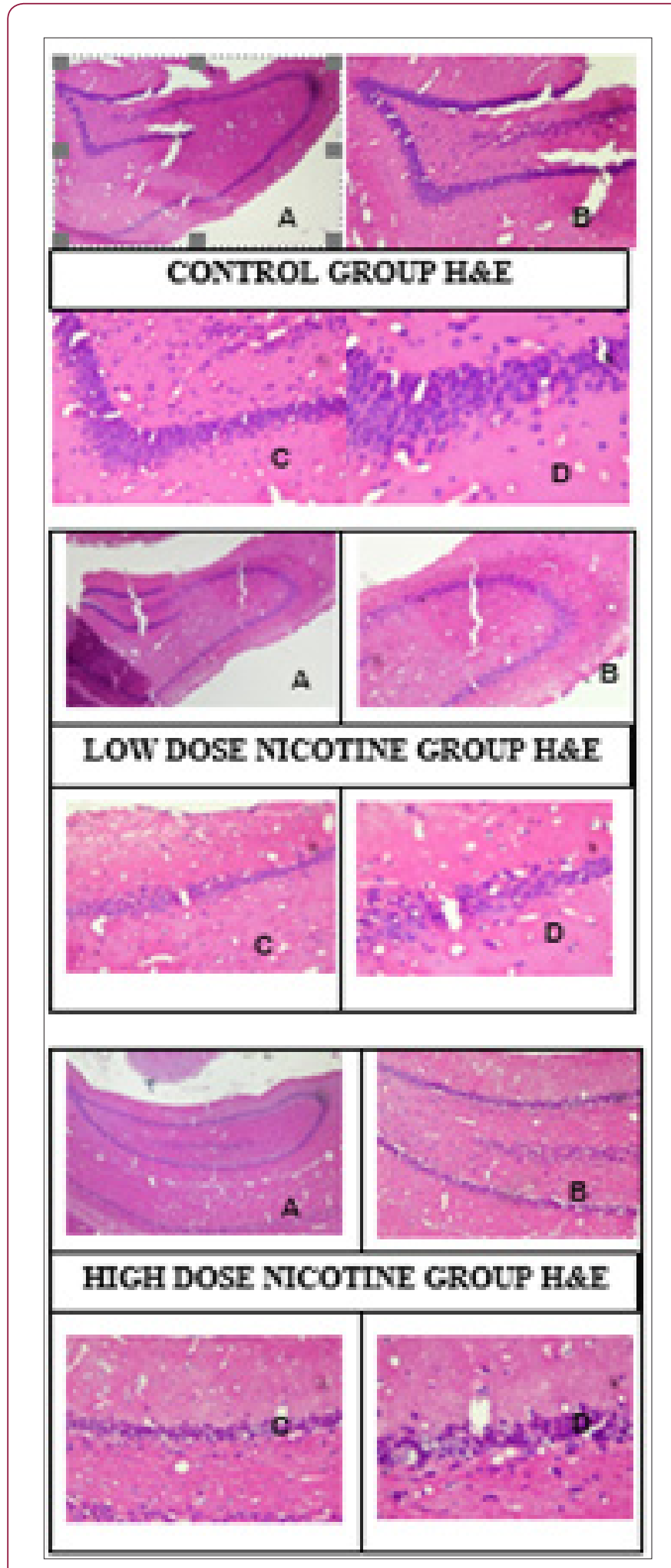

Figure 1: Hipocampus samples of study groups in parafin blockes. As the nicotine dose increased, a decrease in the number of neurons and shrinkage of cells and chromatolysis were observed. X40 (A), X100 (B), X200 (C), X400 (D). 


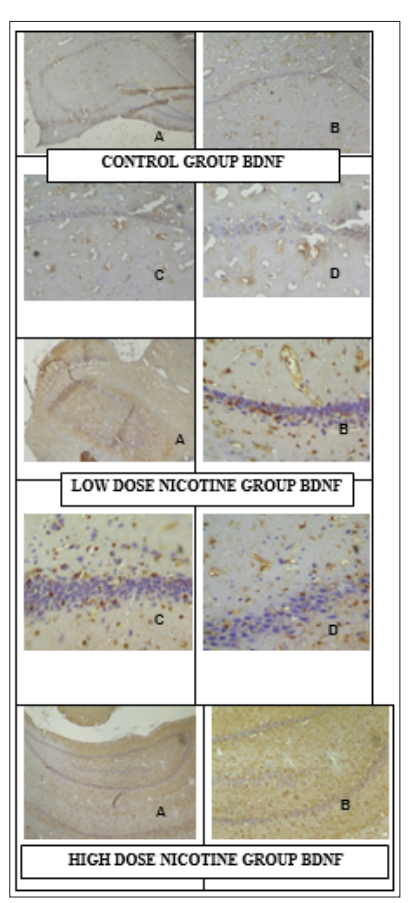

Figure 2: Micrograph of control, $\mathrm{N}$ and $\mathrm{S}$ group's neurons BDNF immunreactivities. X40 (A), X100 (B), X200 (C), X400 (D). The number of BDNF positive stained cells in hippocampus and ventricle was significantly higher than control groups.

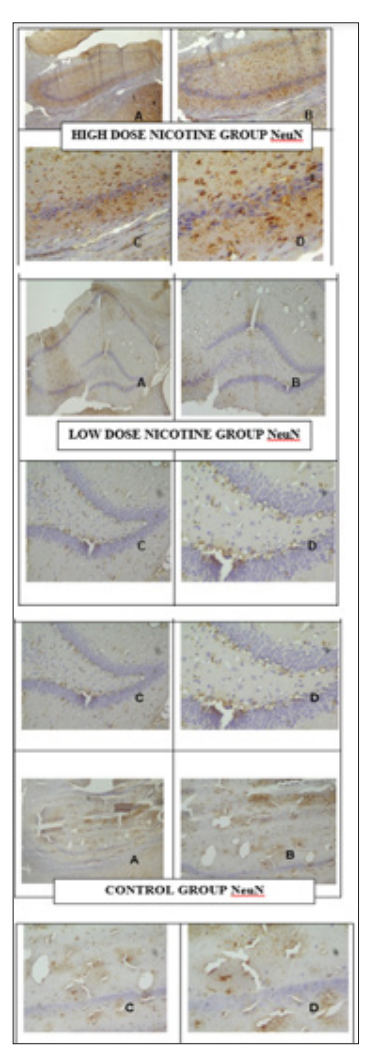

Figure 3: Micrograph of control, $N$ and $S$ group's neurons BDNF immunreactivities. X40 (A), X100 (B), X200 (C), X400 (D). The number of positive stained cells with S100 immunreactivities in nicotin group's neuroglial cells was higher than the control group.
Table 1: Immunreactivity scores of the study groups.

\begin{tabular}{|c|c|c|c|}
\hline & GROUP 1 - control & GROUP 2 - LDN & GROUP 3 - HDN \\
\hline Anti-BDNF & $+/++$ & $++/+++$ & $++/+++$ \\
\hline Anti-NeuN & $+/++$ & ++ & ++ \\
\hline Anti-S-100 & $++/+++$ & +++ & +++ \\
\hline
\end{tabular}

\section{Discussion}

In this study, the effects of pre/postnatal nicotine administration on neurogenesis was investigated. For this purpose; BDNF, NeuN and S100 protein immunoreactivities were analyzed by indirect immunohistochemical methods. When Joseph Altman showed the cell proliferation in adult hippocampus and bulbus olfactories, the studies on neurogenesis were accelerated [10]. Neurogenesis refers to the birth of neurons. In other words, it means as a process of producing neurons from neural stem cells. Neurogenesis has been implicated in the most prenatal period [11]. BDNF expression in the brains of laboratory animals was increased by behavioural interventions such as exercise and training /learning [12]. In this study new neurons and BDNF immunoreactivities of neurons in the hippocampus were found to be increased via nicotine administration. BDNF is a member of neurotrophins and it is widely expressed in the hippocampus, septum, cortex, and in adrenergic brainstem nuclei [13]. The dependency behaviors associated with the effect of addictive drugs are regulated by the change in BDNF concentration [14]. BDNF is important in regulating cell survival and prognosis. The results of Kenny et al. showed that acute administration of nicotine decreases BDNF level whereas chronic nicotine increases BDNF levels in the hippocampus [15].

Additionally,long-termlowandhighdosenicotineadministration increased the neurotrophic factors in rat hippocampus. According to these findings, we thought that nicotine might be effective in adult neurogenesis. NeuN immunoreactivities were increased in the nicotine group. It is suggested that this neuron-specific antigen is an early marker of neuronal differentiation [16]. The hippocampal formation was processed for immunohistochemical staining of NeuN. Nicotine caused to a decrease in the number of NeuN (+) cells dose-dependently [17]. In contrast, Nagai $\mathrm{T}$ et al. showed that nicotine increased NeuN protein immunoreactivity in the hippocampal neurons [18]. Dentate gyrus has important functions in learning, memory and adult neurogenesis. The previous studies showing that Dentate gyrus proliferation is unaltered by short-term nicotine administration and the later stages neurogenesis may not be affected because of the lack of neurogenic effects [19].

S-100 protein is a dimeric, acidic calcium-binding protein which is a major component of the cytosol, particularly in astroglial cells. S100 protein has two subunits, S-100 A and S-100 B. S-100 B protein has regulatory functions on cell shape, growth, differentiation, and energy metabolism. S100 protein is a major component of astroglial cells. Upon injury to nerve cells within the central nervous system, astrocytes fill up the space to form a glial scar, repairing the area and replacing the CNS cells that cannot regenerate so, nicotine might repair the nervous system by the indirect way. $S$ $100 \mathrm{~B}$ protein levels are increased with acute cerebral damage [20]. Kenangil G et al. suggested that serum S-100 B measurement can 
be used as an early marker of brain damage [21]. In our study, anti S 100 immunoreactivities were higher in the nicotine group than the control group. It has been recently shown that nicotine was affecting memory and learning during adolescence. Considering the role of the hippocampus in both memory and learning, we investigated whether adolescent nicotine administration elicits apoptotic cell death and whether this results in neuronal and/or glial density alterations in the hippocampus [22].

In Alzheimer's disease, the hippocampus is one of the first regions of the brain suffering from damage; memory problems and disorientation which appear frequently among the first symptoms. Damage to the hippocampus can also result from oxygen starvation (hypoxia), encephalitis, or medial temporal lobe epilepsy.

\section{Conclusion}

Nicotine, the psychoactive ingredient in tobacco, can be neuroprotective but the mechanism is not exactly known. Nicotine administration can increase the expression of neurotrophic factors and growth factors. These factors can contribute to nicotine's neuroprotective effects [22]. Nicotine exposure can enrich the reduced number of neurons or intraneuronal connections which are decreasing with aging so it can prevent or delay dementia [23]. The role of nicotine in neurodegenerative disorders is still incompletely understood, but it is suggested that progression of these diseases is related to oxidative stress, resulting from either a decrease in antioxidant levels or an increase in reactive oxygen species or reactive nitrogen species. Nicotine has both antioxidant and prooxidant effects [24]. More clinical studies are needed in this situation.

\section{References}

1. Winzer Serhan UH (2008) Long-term consequences of maternal smoking and developmental chronic nicotine exposure. Front Biosci 13: 636-649.

2. Dwyer J, McQuown SC, Leslie FM (2009) The dynamic effects of nicotine on the developing brain. Pharmacol Ther 122(2): 125-139.

3. Coleman T, Britton J, Thornton J (2004) Nicotine replacement therapy in pregnancy. BMJ 328: 965-966.

4. Chistyakov V, Patkina N, Tammimäki A, Talka R, Salminen O, et al. (2010) Nicotine exposure throughout early development promotes nicotine self-administration in adolescent mice and induces long-lasting behavioural changes. Eur J Pharmacol 640: 87-93.

5. Marty S, Berninger B, Carroll P, Thoenen H (1996) GABAergic stimulation regulates the phenotype of hippocampal interneurons through the regulation of brain-derived neurotrophic factor. Neuron 16: 565-570.

6. Rivera C, Hong Li, Judith Thomas Crusells, Hannele Lahtinen, Tero Viitanen, et al. (2002) BDNF-induced TrkB activation down-regulates the $\mathrm{K}^{+}-\mathrm{Cl}^{-}$cotransporter $\mathrm{KCC} 2$ and impairs neuronal $\mathrm{Cl}-$ extrusion. J Cell Biol 159: 747-752.
7. Gusel'nikova V, Korzhevskiy D (2015) NeuN as a neuronal nuclear antigen and neuron differentiation marker. Acta Naturae 7(2): 42-47.

8. Sedaghat F, Notopoulos A (2008) S100 protein family and its application in clinical practice. Hippokratia 12(4): 198-204.

9. Donato R, Cannon BR, Sorci G, Riuzzi F, Hsu K, et al. (2013) Functions of S100 proteins. Curr Mol Med 13(1): 24-57.

10. Eriksson PS, Perfilieva E, Bjork Eriksson T, Alborn AM, Nordborg C, et al. (1998) Neurogenesis in the adult human hippocampus. Nat Med 4(11): 1313-1317.

11. Gross CG (2000) Neurogenesis in the adult brain: death of a dogma. Nat Rev Neurosci 1(1): 67-73.

12. Mattson MP (2004) Pathways towards and away from Alzheimer's disease. Nature 430(7000): 631-639.

13. Chao M, Rajagopal R, Lee F (2006) Neurotrophin signaling in health and disease. Clin Sci 110(2): 167-173.

14. Bhang S, Choi S, Ahn J (2010) Changes in plasma brain derived neurotrophic factor levels in smokers after smoking cessation. Neuroscience Letters 468(1): 7-11.

15. Kenny J, File S, Rattray M (2000) Acute nicotine decreases, and chronic nicotine increases the expression of brain-derived neurotrophic factor mRNA in rat hippocampus. Brain Res Mol Brain Res 85(1-2): 234-238.

16. Mullen R, Buck C, Smith AM (1992) A neuronal specific nuclear protein in vertebrates. Development 116(1): 201-211.

17. Shingo AS, Kito $S$ (2005) Effects of nicotine on neurogenesis and plasticity of hippocampal neurons. J Neural Transm 112(11): 1475-1478.

18. Nagai T, Ito M, Nakamichi M, Mizoguchi H, Hiroyuki Kamei, et al. (2006) The rewards of nicotine: Regulation by tissue plasminogen activatorplasmin system through protease activated receptor-1. The Journal of Neuroscience 26(47): 12374 -12383.

19. Mahar I, Bagot R, Davoli M, Miksys S, Rachel Tyndale F, et al. (2012) Developmental Hippocampal Neuroplasticity in a Model of Nicotine Replacement Therapy during Pregnancy and Breastfeeding. Plos One: pp. 7.

20. Schmidt S (1998) S-100 B: Pathogenetic and pathophysiologic significance in neurology. Nervenarzt 69(8): 639-646.

21. Kenangil G, YalçınD, Haklar G, Cacina H, Forta H, et al. (2004) Relation of serum s-100 protein to infarct size and clinical prognosis. Marmara Medical Journal 17: 105-108.

22. Son J, Ursolo H (2009) Chronic neonatal nicotine exposure increases mRNA expression of neurotrophic factors in the postnatal rat hippocampus. Brainresearch: pp. 1-14.

23. Riljak V, Mılotová M, Jandová K, Pokorni J, Langmeier M, et al. (2007) morphological changes in the hippocampus following nicotine and kainic acid administration. Physiol Res 56(5): 641-649.

24. Newman MB, Arendash GW, Shytle RD, Bickford PC, Tighe T, et al. (2002) Nicotine's oxidative and antioxidant properties in CNS. Life Sci 71(24): 2807-2820. 


\section{ISSN: 2574-1241}

DOI: $10.26717 / B J S T R .2019 .13 .002343$

Soycan Mizrak. Biomed J Sci \& Tech Res

(c) (P) This work is licensed under Creative

Submission Link: https://biomedres.us/submit-manuscript.php

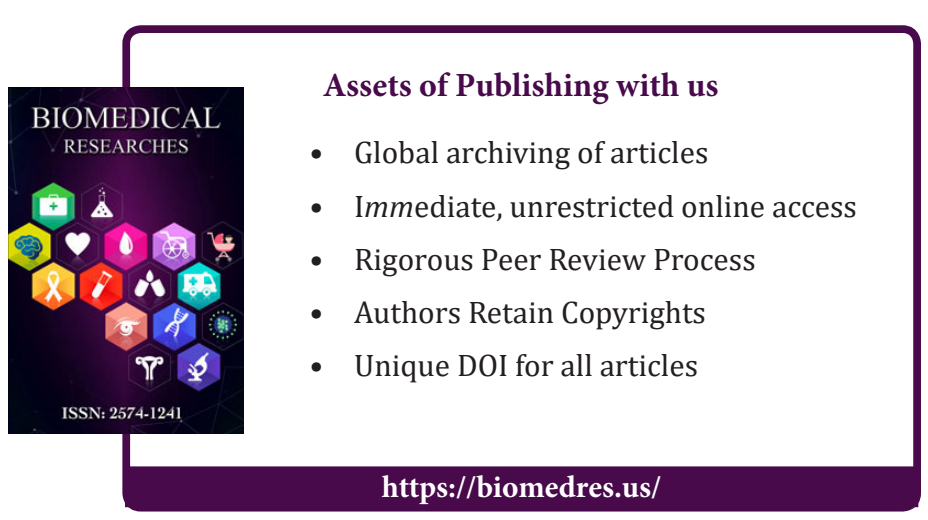

